\title{
Bibliography of Esko Kangas (7 January 1906 -26 December 1992)
}

\author{
Compiled by Ilpo Mannerkoski
}

This bibliography is based on the last curriculum vitae of Esko Kangas, dated 25 November 1992. Most of the references have been checked from the original sources. Only the scientific articles are referred, mostly written in German or English, but also in Finnish with German or English summaries. The abstracts of the lectures held in scientific congresses are also listed. Some articles only in Finnish or Swedish are listed with translation of the title within square brackets. Numerous popular articles published mostly in different forestry magasines have been omitted as well as

\section{1}

Siikakankaan mäntytaimistojen tuhoista. Referat: Über die Schädigungen der Kiefernpflanzenbestände in Siikakangas. — Silva Fennica 17:1-107.

\section{2}

Om några skadeinsekter på tallplantor. [On some pest insects on pine plants]. — Notulae Entomologicae 11:156160.

Tutkimuksia kaasutuhoista Imatran valtionpuistossa. Referat: Untersuchungen über die Rauchschäden im ImatraStaatspark. - Silva Fennica 23:1-37.

1934

Eräitä mielenkiintoisia kovakuoriaislöytöjä. [Some interesting beetlefinds]. — Notulae Entomologicae 14:126127. (Kangas, E. \& Kangas, Y.)

Tutkimuksia Punkaharjun männiköiden hyönteistuhoista. Summary: Untersuchungen über die Insektenschädigungen der Kiefernbestände in Punkaharju. - Communicationes Instituti Forestalis Fenniae 19(7):1-68.

Über entomologische Analysen und ihre Anwendnung. Acta Forestalia Fennica 40(6):1-28.

\section{5}

Die Braunfeckigkeit des Birkenholzes und ihr Urheber Dendromyza (Dizygomyza) betulae n. sp. Vorläufige Mitteilung. Communicationes Instituti Forestalis Fenniae 22(1):1-31. most obituaries and biographies. In Annales Entomologici Fennici (AEF) Esko Kangas published 18 annual reports of the Entomological Society of Finland (ESF) and during 11 years the abstracts of the ESF meetings. Besides these he wrote to AEF over 30 book reviews, 10 reports of the ESF summer excursions and some other articles. In the meetings of ESF Kangas presented about 430 communications reported in Annales Entomologici Fennici (1935-1976), Notulae Entomologicae (1977-1989) and Entomologica Fennica (1991-1992).

Käytännöllinen imuri keräilyä varten. Referat: Ein neuer Exhaustor-Typ. - Annales Entomologici Fennici 1:65-66.

Lyctus planicollis Le Conte varastotuholaisena Suomessa. Summary: Lyctus planicollis Le conte, a new stock-destroyer in Finland. - Annales Entomologici Fennici 1:23-27.

Siikakankaan maakiitäjäisfaunasta. Referat: Über die Carabidenfauna von Siikakangas. - Annales Entomologici Fennici 1:63-64.

Zur Kenntnis der Larven der Pissodes-Arten Finnlands. Communicationes Instituti Forestalis Fenniae 20(3):1-25.

1936

Henoticus germanicus Reitt. als Vertreter einer neuen Cryptophagidengattung, Coniophthalma n. gen. (Col.). Annales Entomologici Fennici 2:67-73.

Über die Braunfleckigkeit der Laubhölzer. — Opuscula Entomologica 1:88-89.

Über Hymenorus doublieri Muls. und H. avajewi Sem. (Col., Alleculidae). - Annales Entomologici Fennici 2:173-178.

1937

Ein Fall von anomaler Fühlerbildung bei Cimbex connata Schrk (Hym., Tenthredinidae). — Annales Entomologici Fennici 3:150-153.

Ein interessanter Fund von Bembidion stephensi Crotch (Col., Carabidae) aus Finnland. - Annales Entomologici Fennici 3:156-160. 
Ein neuer Fall von anomaler Fühlerbildung bei Cimbex (Hym., Tenthredinidae). - Annales Entomologici Fennici 3:225-226.

Tutkimuksia mäntytaimistotuhoista ja niiden merkityksestä. Referat: Untersuchungen über die in Kiefernpflanzbestände auftretenden Schäden und ihre Bedeutung. - Communicationes Instituti Forestalis Fenniae 24(1):1-237.

Über die Braunfeckigkeit der Laubhölzer. - Annales Entomologici Fennici 3:33-39.

Zwei neue hochnordische Bledius-Arten (Col., Staphylinidae) aus Finnland. - Annales Entomologici Fennici 3:185-192.

\section{8}

Revision der finnischen Arten der Gattung Oligota Mann. (Col., Staphylinidae). - Annales Entomologici Fennici $4: 201-212$.

Zur Biologie und Verbreitung der Pissodes-Arten (Col., Curculionidae) Finnlands. - Annales Entomologici Fennici 4:1-20, 73-98.

1939

Über die Wiederstandsfähigkeit der Fichte gegen Angriffe von Dendroctonus micans. - VII Internationalen Kongress für Entomologie, Berlin 1938, Verhandlungen, Band III: 1990-2003.

\section{0}

Aspens avtorkning belyst av entomologiska analyser. [The dying of aspens described by entomological analyses]. Entomologiske Meddelerser 22:32-33.

Kuusen käpytuhot ja siemensato v. 1937. Referat: Zapfenschäden und Samenertrag bei der Fichte im J. 1937. Communicationes Instituti Forestalis Fenniae 29(2):1-38.

Tuloksia Pohjankankaan ja Hämeenkankaan metsänviljelyksistä. Referat: Ergebnisse der Waldkulturen auf den Heiden Pohjankangas und Hämeenkangas. - Acta Forestalia Fennica 49(4):1-64.

Über die Larve und die Lebensweise von Nothorrhina muricata Dalm. (Col., Cerambycidae). - Annales Entomologici Fennici 6:71-77.

Zur Biologie und forstlichen Bedeutung von Callimome azureum Boh. (Hym., Chalcididae). - Annales Entomologici Fennici 6:140-154. (Kangas, E. \& Lovászy, P.)

Zur Biologie von Trypophloeus alni Lindem. (Col., Scolytidae). - Annales Entomologici Fennici 6:41-50.

1941

Agrilus ater L., als Espenschädling in Finnland. - Zeitschrift für Angewandte Entomologie 28:359-365.

Beitrag zur Biologie und Gradation von Diprion sertifer Geoffr. (Hym., Tenthredinidae). - Annales Entomologici Fennici 7:1-31.
Zur Biologie und Verbreitung der Pissodes-Arten (Col., Curculionidae) Finnlands. Nachtrag. - Annales Entomologici Fennici 7:91-92.

1942

Bemerkungen über zwei in Finnland als Waldschädlinge neue Blattwespen. - Annales Entomologici Fennici 8:221223.

Einige Resultate von Parasitwespenzüchtungen. - Annales Entomologici Fennici 8:247-248.

Forstentomologische Studien an der Espe. - Annales Entomologici Fennici 8:49-71.

Forstentomologische Studien an einigen Laubhölzern. Annales Entomologici Fennici 8:142-163.

Karistuslämmön vaikutuksesta männyn siemenen karisemiseen ja itämiseen. Referat: Über den Einfluss der Darrhitze auf die Ausklengung und Keimung des Kiefernsamens. - Acta Forestalia Fennica 50(14):1-42.

Professori Uunio Saalas 60-vuotias. Professor Uunio Saalas zum sechzigsten Geburtstag. - Annales Entomologici Fennici 8:1-10.

1943

Cantharis lateralis L. (Col., Cantharidae) Äänisen Karjalasta. Referat: Cantharis lateralis L. (Col., Cantharidae) aus Äänis- (Onega-) Karelien. - Annales Entomologici Fennici 9:51-52.

Heterogenea asella Schiff. (Lep., Cochlidiidae) tavattu Suomesta. Referat: Heterogenea asella Shiff. (Lep., Cochlidiidae) in Finnland gefunden. - Annales Entomologici Fennici 9:215-216.

Pegohylemyia anthracina Czerny (Dipt., Muscidae) als Zapfenschädling an der Fichte. - Annales Entomologici Fennici 9:215-216. (Kangas, E. \& Leskinen, K.)

1944

Biologische Beobachtungen und Züchtungsversuche an einigen Tenthrediniden (Hym.). - Annales Entomologici Fennici 10:172-181.

Über die Lebensweise und die Larve von Xylophilus cruentatus Gyll. (Col., Eucnemidae). - Annales Entomologici Fennici 10:7-16. (Kangas, E. \& Kangas, J.)

1945

Biologische Beobachtungen und Züchtungsversuche an einigen Tenthrediniden (Hym.). II. - Annales Entomologici Fennici 11:150-156.

Eine Neue Megastigmus-Art (Hym., Chalcididae) als Samenschädling an Abies sibirica. - Annales Entomologici Fennici 11:177-184.

Ein Vertreter der sibirischen Coccinellidenfauna (Col.) in Finnland. Harmonia axyrides Pall. ssp. 19-signata Fald. ab. punctum-suturae n. ab. - Annales Entomologici Fennici 11:63-67. 
Katsaus Suomen Hyönteistieteellisen Seuran 10-vuotisvaiheisiin. Referat: Zum zehnjährigen Bestehen der Finnischen Entomologischen Gesellschaft. - Annales Entomologici Fennici 11:1-13.

1946

Biologische Beobachtungen und Züchtungsversuche an einigen Tenthrediniden (Hym.). III. - Annales Entomologici Fennici 12:14-19.

Kuusikoiden kuivumisesta metsätuho- ja metsänhoidollisena kysymyksenä. Referat: Über die Vertrocknung der Fichtenbestände als Waldkrankheit- und Waldbaufrage. - Acta Forestalia Fennica 52(5):1-192.

Über die Gattung Abia Leach (Hym., Tenthredinidae) im Lichte ihrer europäischen Arten. - Annales Entomologici Fennici 12:77-122.

\section{7}

Beobachtungen über Lyctus planicollis Le Conte in Finnland. - Annales Entomologici Fennici 13:55-58.

Studien über die Agrilus-Larven (Col., Buprestidae). Annales Entomologici Fennici 13:104-113.

Kovakuoriaisfaunamme erikoisuuksia luonnonsuojelun kannalta. [Peculiarities of our coleopterous fauna from the viewpoint of nature protection]. - Suomen Luonto 6:4555.

\section{8}

VII. pohjoismainen hyönteistieteilijäin kokous Helsingissä elokuun 5.-7. p:nä 1947. [The VII Nordic Congress of Entomology, 5-7 August 1947]. — Annales Entomologici Fennici 14 suppl.: 9-39.

Die Dendromyza-Arten von Eberesche, Espe und Salweide und ihre Biologie. - Annales Entomologici Fennici 14 Suppl.: 106-117.

Om Blediusarternas genitalier. [On the genitals of the Bledius-species]. - Annales Entomologici Fennici 14 Suppl.:117-118.

Syneta betulae F. (C., Chrysomelidae) als Fichtenschädling in Nordnorwegen. - Annales Entomologici Fennici 14:1922.

\section{9}

Hirven metsässä aikaansaamat tuhot ja niiden metsätaloudellinen merkitys. Summary: On the damage to the forests caused by the moose, and its significance in the economy of the forests. - Suomen Riista 4:62-90.

Xylechinus pilosus Ratz. und Polygraphus poligraphus L. (Col., Scolytidae) an der Kiefer. - Annales Entomologici Fennici 15:168-174.

\section{0}

Die Primärität und Sekundärität als Eigenschaften der Schädlinge. - VIII International Congress of Entomology, Stockholm 1948, Proceedings s. 792-798.
Über die Objektwahl des Schadenurhebers bei einigen Waldschäden. Theoretische und Materialbetrachtung. Annales Entomologici Fennici 16:145-177.

1951

Die finnischen Euplectus-Arten (Col., Pselaphidae). Annales Entomologici Fennici 17:136-148.

\section{2}

Die Larve von Leptura maculicornis DeG. (Col., Cerambycidae), nebst Erörterungen über die Biologie einiger Leptura-Arten. - Annales Entomologici Fennici 18:75-80.

Eine neue Art der Gattung Leptusa Kraatz, L. saalasi n. sp. (Col., Staphylinidae) aus Finnland. - Annales Entomologici Fennici 18:4-8.

Maannousemasienen (Polyporus annosus Fr.) esiintymisestä, tartunnasta ja tuhoista Suomessa. Referat: Über Auftreten, Infektion und Schäden des Wurzelschwamms (Polyporus annosus Fr.) in Finnland. - Communicationes Instituti Forestalis Fenniae 40(33):1-34.

Über die Brutstättenwahl von Dendroctonus micans Kug. (Col., Scolytidae) auf Fichten. - Annales Entomologici Fennici 18:154-170.

1953

On population regulation of forest insects living in tree rind in Finland. - IX International Congress of Entomology, Amsterdam 1952, Transactions, vol. 2:224-228.

Zur ausbreitung des Carabus Cancellatus Ill. (Col., Carabidae) in jüngster Zeit in Finnland. - Annales Entomologici Fennici 19:175-181.

\section{4}

On the possibility of pests being conveyed in export timber. Survey of biological requirements. - Acta Forestalia Fennica 61(23):1-11.

Puun kuoressa elävien hyönteisten gradaatiotekijöistä. [On the gradation factors affecting insects living in tree rind). — Luonnon Tutkija 58:97-104.

\section{5}

Dendromyza cerasiferae n.sp. (Dipt., Agromyzidae) from England. - Annales Entomologici Fennici 21:165-170.

Die orientierung der rindenbewohnenden Forstschädlinge. Zur Metodik und forstlichen Bedeutung ihrer Untersuchung. - Verhandlungen der Deutschen Gesellschaft für Angewandte Entomologie E.V. 1954, Berlin, s. 47-51.

Pelecotoma fennica Payk. (Col., Rhipiphoridae) lajin esiintymisestä Fennoskandiassa. Referat: Über das Vorkommen von Pelecotoma fennica Payk. (Col., Rhipiphoridae) in Fennoskandien. - Annales Entomologici Fennici 21:12-20. (Kangas, E. \& Kangas, J.)

Tuhohyönteisten kohteenvalinta sovelletun entomologian probleemana. [The choice of host in insect pests as a problem of applied entomology.] — Luonnon Tutkija 59:68-72. 
Weitere Beiträge zur Kenntnis der Ausbreitungsdynamik von Carabus cancellatus Ill. (Col., Carabidae). - Archivum Societatis Zoologicae Botanicae Fennicae Vanamo 9:113115.

\section{6}

Über das Vorkommen des Hymenorus doublieri Muls. (Col., Alleculidae) in Finnland. - Annales Entomologici Fennici 22:154-156.

\section{7}

Eine neue Gnypeta-Art (Col., Staphylinidae) aus Sibirien. - Annales Entomologici Fennici 23:85-86.

Über die Larve, Puppe und die Biologie von Pissodes gyllenhali Gyll. (Col., Curculionidae). - Zeitschrift für Angewandte Entomologie 41:153-158.

\section{8}

Dritter Beitrag zur Kenntnis der Ausbreitungsdynamik von Carabus cancellatus Ill. (Col., Carabidae). - Annales Entomologici Fennici 24:181-186.

Über die forstzoologischen Probleme in Finnland. Anzeiger für Schädlingskunde 31:161-165.

Über die jüngsten Untersuchungen auf dem Gebiete der forst- und landwirtschaftlichen Zoologie in Finnland. Zeitschrift für Pflanzenkrankheiten und Pflanzenschutz 65:411-416. (Kangas, E. \& Kanervo, V.)

Über die rotbunten Arten der Gattung Badister Clair. (Col., Carabidae) in Finnland. - Annales Entomologici Fennici 24:131-141.

\section{9}

Eine neue Art der Gattung Hallomenus Panz. (Col., Serropalpidae) aus Finnland. - Annales Entomologici Fennici 25:187-192.

Lisääntymismateriaalin valinta sidotusti elävien metsätuholaisten runsautta säätelevänä tekijänä [The choice of breeding material as a factor regulating the abundance of Forest pests bound to their host tree]. - Suomalainen tiedeakatemia. Esitelmät ja pöytäkirjat 1958: 203-210.

Silvicultural research in Finland from 1909 to 1959. Acta forestalia Fennica 70(4):1-28. (Mikola, P., Kangas, E. \& Heikurainen, L.)

Som skogsarter förekommande träinsekternas övergång till anthropochorer. [The change of forest insects living in wood to anthropochores]. - Notulae Entomologicae 39:85.

Systematische Untersuchungen über die Larven der Hylobius piceus DeG., abietis L. und pinastri Gyll. (Col., Curculionidae). - Annales Entomologici Fennici 25:110-118.

Veränderung von als Waldarten vorkommenden Holzinsekten in anthropochoren. - Beiträge zur Vorratsschutzforschung. Festschrift Friedrich Zacher. Berlin. s. 39-41.

\section{0}

Über das Vorkommen der Monochamus-Arten (Col., Cerambycidae) und ihre Bekämpfungsmöglichkeiten in
Finnland. - Annales Entomologici Fennici 26:263-272. (Kangas, E. \& Salonen, K.)

Über die Biologie und die Larve von Abia candens Kon. (Hym., Cimbicidae). - Annales Entomologici Fennici 26:51-56.

\section{1}

Aus der Tätigkeit der Finnischen entomologischen Gesellschaft 1935-1960. — Anzeiger für Schädlingskunde 34:9192.

Hyönteisten, erityisesti tuholaisina esiintyvien lajien reagoinnista voimistuvaan inhimilliseen kulttuuriin. Summary: The impact of human culture on insects, and especially on insect pests. - Fennia 85:100-105.

Über die Atomaria-arten (Col., Cryptophagidae) Finnlands. - Annales Entomologici Fennici 27:112-118.

Waldschädlingsprobleme, hervorgerufen durch die jüngste Entwicklung auf dem Gebiete des Waldbaues und der Forstbenutzung in Finnland. - XI International Kongress für Entomologie, Wien 1960. Verhandlungen, band 2:167172.

Zur Biologie von Pamphilius albopictus Thoms. (Hym., Pamphiliidae). — Annales Entomologici Fennici 27:69-72.

1962

Professor Dr. Uunio Saalas 80 Jahre. - Zeitschrift für Angewandte Entomologie 50:247-248.

Studies on Hylobius abietis L. 1: Development and life cycle in the Nordic countries. - Acta Entomologica Fennica 17. 107 p. (Bejer-Petersen, B., Juutinen, P., Kangas, E., Bakke, A., Butovitsch, V., Eidman, H., Heqvist, K.J. \& Lekander, B.)

Über Krummstämmigkeit und Verzweigung der finnischen Kiefer. - Communicationes Instituti Forestalis Fenniae 55(15):1-16.

Über das schädliche Auftreten der Diprion-Arten (Hym., Diprionidae) in finnischen Kieferbeständen in diesem Jahrhundert. - Zeitschrift für Angewandte Entomologie 50:188-194.

\section{3}

Die Larve von Pamphilius albopictus Thoms. (Hym., Pamphiliidae). - Annales Entomologici Fennici 29:267269. (Kangas, E. \& Kangas, J.)

Die Waldwirtschaft im finnischen Lappland und ihre Borkenkäferprobleme. - IUFRO Discussion Group Population Dynamics 1963. 6 p.

Tallens reagering till skador förorsakade av Evetria resinella L. (Lep.). [The reaction of pine to damage caused by Evetria resinella L. (Lep.)] — Entomologiske Meddelerser 32:67-70.

1964

Forest insect control, silvicultural measures. - FAO/ IUFRO, Symposium on international dangerous forest diseases and insects, Oxford 1964. 8 p. 


\section{5}

Orientation of Blastophagus piniperda L. (Col., Scolytidae) to its breeding material. Attractant effect of $\alpha$-terpineol isolated from pine rind. - Annales Entomologici Fennici 31:61-73. (Kangas, E., Perttunen, V., Oksanen, H. \& Rinne, M.)

Über die Kollektivart Conosoma testaceum Fabr. (Col., Staphylinidae) in Finnland. - Annales Entomologici Fennici 31:111-114.

Weitere biologische Beobachtungen und Neubeschreibung der Larve von Pamphilius albopictus Thoms. (Hym., Pamphiliidae). - Annales Entomologici Fennici 31:31-37. (Kangas, E. \& Kangas, J.)

1967

Laboratory experiments on the olfactory orientation of Blastophagus piniperda L. (Coleoptera, Scolytidae) to substances isolated from pine rind. - Acta Entomologica Fennica 22. 87 p. (Kangas, E., Perttunen, V., Oksanen, H. \& Rinne, M.)

Notes on the arthropod fauna of Spitsbergen. I: 5. Identification of the Coleoptera collected by the Finnish Spitsbergen expeditions. - Annales Entomologici Fennici $33: 41-43$.

Studies on the olfactory stimuli guiding the bark beetle Blastophagus piniperda L. (Coleoptera, Scolytidae) to its host tree. - Annales Entomologici Fennici 33:181-211. (Kangas, E., Perttunen, V. \& Oksanen, H.)

\section{8}

Användning av insekticider i Finland på skogssidan under de senaste 10 åren. Summary: Insecticides in Finnish forestry. - Opuscula Entomologica 33:46-49.

The chemical composition of the breeding material of Blastophagus piniperda L. (Col., Scolytidae), and its significance in the olfactory orientation of this species. Annales Entomologici Fennici 34:1-13. (Oksanen, H., Kangas, E. \& Perttunen, V.)

The mechanisms by which Blastophagus piniperda L. (Col., Scolytidae) reacts to the odour of an attractant fraction isolated from pine phloem. - Annales Entomologici Fennici 34:205-222. (Perttunen, V., Kangas, E. \& Oksanen, H.)

Über die Orientierungsmechanismen der Borkenkäfer auf ihr Fortpflanzungsmaterial. - Anzeiger für Schädlingskunde 41:177-180.

Über Helophorus walkeri Sharp und flavipes Fabr. (Col., Hydrophilidae) in Finnland. - Annales Entomologici Fennici 34:38-41.

Untersuchungen über die Einwirkung der die Orientierung der Borkenkäfer leitenden chemischen Verbindungen im Baum. - Zeitschrift für Angewandte Entomologie 61:353364.

1970

Adistemia watsoni Woll. (Col., Lathridiidae) in Finnland gefunden. - Annales Entomologici Fennici 36:122 (Kangas, E. \& Koponen, M.)
Aspects of the external and internal factors affecting the olfactory orientation of Blastophagus piniperda (Coleoptera: Scolytidae). - Contributions from Boyce Thompson Institute 24:293-298. (Perttunen, V., Oksanen, H. \& Kangas, E.)

Om märgborreskadornas biologiska bakgrund. [On the backround of the damage caused by the pine shoot beetles]. - 12. Nordiska skogskongressen. Nordiskt skogsbruk av i dag, p. 359-363.

Responses of Blastophagus piniperda L. (Col., Scolytidae) to trans-verbenol, cisverbenol, and verbenone, known to be population pheromones of some American bark beetles. - Annales Entomologici Fennici 36:75-83. (Kangas, E., Oksanen, H. \& Perttunen, V.)

Responses of Blastophagus minor Hart. (Col., Scolytidae) to the pine phloem fraction known to be attractant to Blastophagus piniperda L. - Annales Entomologici Fennici 36:120-122. (Kangas, E., Perttunen, V. \& Oksanen, H.)

Studies on the chemical factors involved in the olfactory orientation of Blastophagus piniperda (Coleoptera: Scolytidae). - Contributions from Boyce Thompson Institute 24:299-303. (Oksanen, H., Perttunen, V. \& Kangas, E.)

Thinobius praetor Smet. in Fennoskandien. - Annales Entomologici Fennici 36:122-123.

\section{1}

15. Pohjoismainen hyönteistieteilijäin kongressi Helsingissä 27-29 heinäkuuta 1970. Summary: Fifteenth Nordic Congress of Entomology in Helsinki 27-29 July 1970. — Acta Entomologica Fennica 28:7-22.

Physical and chemical stimuli affecting the behaviour of Blastophagus piniperda L. and B. minor Hart. (Col., Scolytidae). - Acta Entomologida Fennica 28:120-126. (Kangas, E., Perttunen, V. \& Oksanen, H.)

Uunio Saalas, memorial address, delivered December 12, 1969. - Proceedings of the Finnish Academy of Science 1969:98-106.

Zum Vorkommen von Pityophthorus micrographus L. (Col., Scolytidae) an der Kiefer. - Annales Entomologici Fennici 37:27-30.

1972

A mechanism accounting for the orientation of bark beetles to their breeding material. - XIII International Congress of Entomology, Moscow 1968. Proceedings, vol. 3:49-50. (Kangas, E. \& Perttunen, V.)

Stenus pubescens Steph. in Finnland. - Annales Entomologici Fennici 38:92.

1973

Note on the arthropod fauna of Spitsbergen. III: 18. Über die Coleopterenfauna in Spitzbergen. - Annales Entomologici Fennici 39:68-70.

Olfactory responses of Hylobius abietis L. (Col., Curculionidae) to substances naturally present in pine phloem or their synthetic counterparts. - Annales Entomologici Fennici 39:40-45. (Selander, J., Kangas, E., Perttunen, V. \& Oksanen, H.) 
1974

Olfactory behaviour of Hylobius abietis L. (Col., Curculionidae). I: Response to several terpenoid fractions isolated from Scots pine phloem. - Annales Entomologici Fennici 40:108-115. (Selander, J., Kalo, P., Kangas, E. \& Perttunen, V.)

The chemical composition of volatiles produced by the host of Hylobius abietis L. (Col., Curculionidae). I: The terpene composition of pine seedlings. - Annales Entomologici Fennici 40:86-95. (Kalo, P., Selander, J., Kangas, E. \& Perttunen, V.)

1975

Über die in der Orienterungsphase der Borkenkäfer auftretenden Prinzipien. - Zeitschrift für Angewandte Entomologie 77:317-325.

\section{6}

Die fennoskandischen Arten der Gattung Miarus Steph. (Col., Curculionidae). - Annales Entomologici Fennici 42:69-83.

The distribution of bark beetles in the Nordic countries. Acta Entomologica Fennica 32. 36 + 66 p. (Lekander, B., Bejer-Petersen, B., Kangas, E. \& Bakke, A.)

Über die an Cruciferae lebenden Ceuthorrhynchus-Arten (Col., Curculionidae) Finnlands. - Annales agriculturae Fenniae 15:72-80.

Über die schwarzen Mordellistena-Arten (Col., Mordellidae) in Finnland. - Annales Entomologici Fennici 42:177-182.

\section{8}

Mordellistena carinthiaca neu für Finnland und Schweden (Coleoptera, Mordellidae). - Notulae Entomologicae 58:127-128.

Über das Problem Miarus campanulae (Linné) (Coleoptera, Curculionidae). - Annales Entomologici Fennici 44:115120.

1979

Über die Arten der Philonthus varians (Paykull) -Gruppe (Coleoptera, Staphylinidae). - Annales Entomologici Fennici 45:99-102.

1980

Ist Pityophthorus traegardhi (Coleoptera, Scolytidae) ein Forstschädling? - Annales Entomologici Fennici 46:4952.

Merkmale und Verbreitung einer neuen und zweier bekannten Unterarten des laufkäfers Bembidion petrosum (Coleoptera: Carabidae). - Entomologia generalis 6:363365.

Über Miarus portae Solari var. confusus Solari in litt. (Coleoptera, Curculionidae). - Annales Entomologici Fennici 46:111-115.
1981

Über die wechselseitigen Beziehungen zwischen Forstschädlingen und ihren Wirtpflanzen. - Forstarchiv 52:170174.

1982

Uunio Saalas tiedemiehenä ja ihmisenä. 100 vuotta Uunio Saalaan syntymästä. [Uunio Saalas as a scientist and a person. 100 years since the birth of Uunio Saalas]. Luonnon Tutkija 86:152-154.

Über einige Arten der Oligota pusillima (Gravenhorst)Gruppe (Coleoptera, Staphylinidae). - Annales Entomologici Fennici 48:65-70.

1983

Eine neue Oxypoda-Art (Coleoptera, Staphylinidae) aus Finnland. - Annales Entomologici Fennici 49:54-56.

1984

Über das Angriffsvermögen des Scolytus ratzeburgi (Coleoptera, Scolytidae) auf eine Inonotus obliquus-faule Birke. - Annales Entomologici Fennici 50:126-127.

Four Mordellistena species (Coleoptera: Mordellidae) new to Sweden. - Entomologisk Tidskrift 105:99-101. (Kangas, E. \& Rutanen, I.)

1985

Time of oviposotion and its influence on the development of Hylobius abietis (L.) (Col., Curculionidae). - Zeitschrift für Angewandte Entomologie 100:417-421. (Lekander, B., Eidmann, H.H., Bejer, B. \& Kangas, E.)

1986

Eine neue Mordellistena-Art (Coleoptera, Mordellidae). - Annales Entomologici Fennici 52:139-141.

Suomen hyönteistieteellisen seuran 50-vuotishistoria. [The 50-year-history of the Entomological Society of Finland]. Helsinki, 155 p.

1988

Mordellistena dalmatica Ermisch sensu Kangas (1976) (Coleoptera, Mordellidae) eine neue unbeschriebene Art. — Annales Entomologici Fennici 54:89-92.

1989

Untersuchungen über die Weibchen der Gattung Malthodes Kiesenwetter (Coleoptera, Cantharidae). - Annales Entomologici Fennici 55:29-33.

1992

Keidas keskellä kaupunkia. [An oasis within the town]. Biological Reports from the University of Jyväskylä 25:9599.

1993

Identification of females of the Finnish species of Altica Mueller (Coleoptera, Chrysomelidae). - Entomologica Fennica (in press). (Kangas, E. \& Rutanen, I.) 